\title{
Vorstand und Ausschuss ab 1. Jänner 2014
}

\section{$\%$ OGIM \\ Österreichische Gesellschaft für Innere Medizin}

\author{
Vorsitzender \\ Univ.Prof.Dr. Herbert Watzke
}

Stellvertretender Vorsitzender

Univ.Prof.Dr. Ernst Pilger

\section{Generalsekretär}

ao.Univ.Prof.Dr. Markus Peck-Radosavljevic

\section{Kassier}

Prim.Univ.Doz.Dr. Franz Xaver Roithinger

\section{Fachärzteausbildungsverantwortlicher}

Doz.Dr. Bernhard Angermayr

\section{Bundesfachgruppenobmann für Innere Medizin} in der ÖÄK

Dr. Lothar Fiedler

\section{Vorstände der Universitätskliniken für Innere}

Medizin

o.Univ.Prof.Dr. Gerald Maurer

Univ.Prof.Dr. Gert Mayer

Univ.Prof.Dr. Ernst Pilger

o.Univ.Prof.Dr. Josef Smolen

Univ.Prof.Dr. Christoph Zielinski

\section{Tagungspräsident 2014}

Univ.Prof.Dr. Herbert Watzke

\section{Tagungssekretär 2014}

ao.Univ.Prof.Dr. Florian Thalhammer

\section{Präsidenten der wissenschaftlichen} Gesellschaften der internistischen Additivfächer Prim.Univ.Prof.Dr. Karl Benedetto - Österreichische Gesellschaft für Internistische Sportmedizin und Prävention

ao.Univ.Prof.Dr. Marianne Brodmann - Österreichische Gesellschaft für Internistische Angiologie

Prim.Dr. Gabriele Eberl, MBA - Österreichische Gesellschaft für Rheumatologie und Rehabilitation
ao.Univ.Prof.Dr. Bernd Jilma - Österreichische Pharmakologische Gesellschaft, Abteilung für Klinische Pharmakologie

Univ.Prof.Dr. Michael Joannidis - Österreichische Gesellschaft für Allgemeine und Internistische Intensivmedizin und Notfallmedizin

ao.Univ.Prof.Dr. Wolfgang Petritsch - Österreichische Gesellschaft für Gastroenterologie und Hepatologie

Prim.Dr. Katharina Pils - Österreichische Gesellschaft für Geriatrie und Gerontologie

Univ.Prof.Dr. Alexander Rosenkranz - Österreichische Gesellschaft für Nephrologie

Univ.Prof.Dr. Hellmut Samonigg - Österreichische Gesellschaft für Hämatologie und Onkologie

Prim.Univ.Doz.Dr. Vinzenz Stepan - Österreichische Gesellschaft für Endokrinologie und Stoffwechsel

Univ.Prof.Dr. Franz Weidinger - Österreichische Kardiologische Gesellschaft

Univ.Prof.Dr. Günter Weiss - Österreichische Gesellschaft für Infektionskrankheiten und Tropenmedizin

\section{Österreichische Gesellschaft für Pneumologie OA Dr. Sylvia Hartl}

\section{Fortbildungsverantwortlicher} ao.Univ.Prof.Dr. Florian Thalhammer

\section{Gewählte Ausschussmitglieder auf Vorschlag des Vorstandes \\ Dr. Susanne Biowski-Frotz \\ Prim.Univ.Prof.Dr. Meinhard Kneussl \\ Prim.Prof.Dr. Kurt Lenz \\ Chefärztin Prof.Dr. Petra-Maria Schumm-Dräger}

\section{Regionale Fortbildungsbeauftragte}

Univ.Prof.Dr. Martin Clodi

Univ.Prof.Dr. Renate Koppensteiner o.Univ.Prof.Dr. Otmar Pachinger

Univ.Prof.Dr. Ernst Pilger

Priv.Doz.OA Dr. Gernot W. Wolkersdörfer 